\title{
Peer-to-peer energy markets: Understanding the values of collective and community trading
}

\author{
Leave Authors Anonymous \\ for Submission \\ City, Country \\ e-mail address
}

\author{
Leave Authors Anonymous \\ for Submission \\ City, Country \\ e-mail address
}

\author{
Leave Authors Anonymous \\ for Submission \\ City, Country \\ e-mail address
}

\begin{abstract}
Peer-to-peer energy-trading platforms (P2P) have the potential to transform the current energy system. However, research is presently scarce on how people would like to participate in, and what would they expect to gain from, such platforms. We address this gap by exploring these questions in the context of the UK energy market. Using a qualitative interview study, we examine how 45 people with an interest in renewable energy understand $\mathrm{P} 2 \mathrm{P}$. We find that the prospective users value the collective benefits of $\mathrm{P} 2 \mathrm{P}$, and understand participation as a mechanism to support social, ecological and economic benefits for communities and larger groups. Drawing on the findings from the interview analysis, we explore broad design characteristics that a prospective $\mathrm{P} 2 \mathrm{P}$ energy trading platform should provide to meet the expectations and concerns voiced by our study participants.
\end{abstract}

\section{Author Keywords}

Peer to peer energy trading platforms; semi-structured interview; sustainability; thematic analysis .

\section{CCS Concepts}

-Human-centered computing $\rightarrow$ User studies; User centered design; Social engineering (social sciences);

\section{INTRODUCTION}

The way that people can participate in the energy market is changing. By 2030 at least $27 \%$ of Europe's energy generation will come from renewable sources [1]. Much of the newly generated renewable energy (RE) will not be produced by large-scale and centralized generating stations, instead it will be produced by local small-scale, geographically-distributed generators ('microgenerators') - including households, community and cooperative schemes - who typically produce electricity for their own consumption, with any excess exported to the grid [49].

Transition to RE sources is motivated not only by their technological and market viability, but more significantly, by the

\footnotetext{
Permission to make digital or hard copies of all or part of this work for personal or classroom use is granted without fee provided that copies are not made or distributed for profit or commercial advantage and that copies bear this notice and the full citation on the first page. Copyrights for components of this work owned by others than the author(s) must be honored. Abstracting with credit is permitted. To copy otherwise, or republish, to post on servers or to redistribute to lists, requires prior specific permission and/or a fee. Request permissions from permissions@ acm.org.

CHI'20, April 25-30, 2020, Honolulu, HI, USA

(C) 2020 Copyright held by the owner/author(s). Publication rights licensed to ACM. ISBN 978-1-4503-6708-0/20/04 ..\$15.00

DOI: https : //doi .org/10.1145/3313831. XXXXXXX
}

pressing decarbonization agenda due to accelerating climate change. Harnessing the locally available, clean energy sources (such as sunlight and wind) for energy generation is a key avenue for the traditionally fossils-based energy systems' decarbonization.

Although these new forms of (micro-)generation present many opportunities for advancing decarbonization process (DP), there are still many challenges to overcome [10]. Such challenges include development of new energy transaction models, which could adequately motivate, compensate and incentivize distributed generation [12].

Peer-to-Peer (P2P) energy trading is one such model that has the potential to address many of these challenges $[5,6,38$, 44]. In the preset paper we consider energy trading to be $\mathrm{P} 2 \mathrm{P}$ if energy is produced by microgenerators with the excess generation (i.e., what is left after the self-consumption needs of the generator are met) sold directly to other (micro)consumers. Thus, the microgenerators are able to freely and continuously buy and sell their excess generation to/from each other; they can leverage dynamic pricing and aggregation of electricity generation, and incentivize consumers to change their electricity consumption to balance supply and demand. $\mathrm{P} 2 \mathrm{P}$ energy trading must be supported with a digital platform where the buying, selling, billing, and reporting takes place. Yet, research is presently scarce on how people would like to participate in $P 2 P$ trading and what would they expect to gain from such platforms [4, 17, 37].

If $\mathrm{P} 2 \mathrm{P}$ energy-trading platforms are to be widely adopted and successful, we need to consider how to create socially, economically, and ecologically sustainable platforms [18]. Given that there are many technologies and system architectures that can underpin $\mathrm{P} 2 \mathrm{P}$ platforms, which provide different opportunities for participation, control, anonymity and transparency [4, 13], it is pertinent to study how prospective users perceive the $\mathrm{P} 2 \mathrm{P}$ energy market, what they would expect from these systems and other platform participants, and the type of participation they would seek. Here we present the first such study.

In this paper we explore how members of the public understand P2P energy trading, including what would motivate their participation in a P2P platform, desired benefits and the types of interactions they wish to have. We also explore how P2P can support users' wholistic engagement with DP and energy sustainability; including how to enable users to aggregate resources to balance electricity supply and demand, how 
to enable widespread participation and support those in fuel poverty. This study is carried out through a series of in-depth interviews with people who have a self-defined interest in RE.

Drawing on the findings from the interview analysis, we explore broad design characteristics that a prospective $\mathrm{P} 2 \mathrm{P}$ energy trading platform should provide to meet the expectations and concerns voiced by our study participants. The key contributions of this paper are both in detailing the perceptions of what prospective users expect a $\mathrm{P} 2 \mathrm{P}$ trading planform to be, and the design characteristics that such perceptions entail.

Below, we briefly outline the state of the current research and practice on P2P energy trading platforms (see Related Work), then present our interview study methodology which, using stories and visual scenario props, engages prospective P2P platform users into discussion (presented in Method section). The interview results are analyzed using thematic analysis (Analysis and P2P Platform Design Implications) and further discussed in the Discussion, where we conclude the paper.

\section{RELATED WORK}

There has been a wealth of devices and technology exploration to promote sustainable lifestyles by supporting people's awareness of their energy consumption and how related behavior may impact the environment [20, 21, 40, 41]. For example, there are tools to support energy awareness and efficiency in the home [23, 24, 32], applications that help users switch to a low-carbon electricity supply [55], and efforts to help users shift consumption to more favorable times [52, 33]. However, most existing research is concerned with energy consumption, personal use and rational choice. In contrast, P2P energytrading systems are designed to support both the consumption and generation of RE, and highlight the social, collective and community dimensions of energy sustainability.

To our knowledge, to date very little research has examined the human dimensions of $\mathrm{P} 2 \mathrm{P}$ energy trading that support widespread use, acceptance and participation in DP. This is somewhat surprising as there are real-world examples of $\mathrm{P} 2 \mathrm{P}$ energy-trading systems in use, such as the Brooklyn Microgrid $[43,56]$, though admittedly these tend to be small-scale, experimental deployments focused on study of the technological and economic viability of the platforms.

The HCI community has started to consider how P2P energytrading platforms can support distributed generation, highlighting opportunities for design. For example, research has demonstrated that there is a demand for local energy, but concerns about sharing location data can be a barrier to a location-based approach [42]. Likewise, evidence shows that user values of autonomy, control and economic equality are prominent in these markets [47].

However, P2P energy markets are still largely unexplored in HCI. In their comprehensive literature review, Pierce and Paulos identified a dearth of HCI research examining smart grid and distributed generation technologies. They outlined opportunities for examining "experiential, behavioral, social, and cultural" dimensions of these technologies, including designing to facilitate a sense of ownership and responsibility over energy, and designing to support norms of community sharing [45]. Despite this theoretical contribution, empirical research has focused on the individual and economic dimensions of trading electricity, so we have limited insight into other user values and interests in this context, and how these platforms can support environmental sustainability. This is important because it is likely that $\mathrm{P} 2 \mathrm{P}$ energy markets are not just used as a resource exchange platform, but rather bound-up with a sense of community and wider social, ecological and emotional benefit (e.g., [45]).

To help understand $\mathrm{P} 2 \mathrm{P}$ energy trading, we conceptualize it as a specific instance of the sharing economy. This includes commercial markets such as Airbnb and Uber, as well as non-profit initiatives like Couchsurfing and timebanks [8, 22, $28,34]$. Existing research examining the sharing economy demonstrates the importance of understanding what motivates users and reflecting these motives within the platform interface. It also highlights the need to support sustainability within P2P markets.

Firstly, research examining the sharing economy demonstrates that users have different motives for participation and can obtain different benefits (e.g., [26, 35, 39]). For example, social benefits are important for the use of non-profit services like timebanking and Couchsurfing; these include developing individual and community capabilities, social relations, social care, self-respect and satisfaction [8, 28]. In contrast, Kameswaran, Cameron, and Dillahunt [29] examined the non-financial benefits of commercial ridesharing services, they demonstrated ways that users gain social and cultural resources from others; including informational and emotional companionship support. However, other research has highlighted the role of basic self-interested and instrumental motives, such as obtaining what is needed at a good price with high convenience, for a variety of sharing applications including transportation services and Airbnb $[28,7,16]$. Significantly, research demonstrates that a mis-match between user motives and the benefits of use can be detrimental for participation, thus it is important to appropriately understand and embody user motives within the interface to create successful platforms $[8,7]$.

Additionally, the field of $\mathrm{HCI}$ has recently adopted a critical approach to explore the equality and social justice implications of the sharing economy (e.g., [15]). On the one hand, this research seeks to understand how to enable everyone to participate: Research tends to demonstrate that low-income households are unable to participate in and benefit from platforms such as Uber and Airbnb, for example due to low digital literacy and restricted payment methods [14, 31] Simultaneously, this work demonstrates the additional challenge that P2P platforms can reflect and reproduce inequality. This has been accompanied by calls for the CHI community to find ways to support "ecological, economic and social sustainability, with the goal to promoting a fairer distribution of goods and labor, ultimately creating a stronger sense of community" [18].

These considerations are particularly relevant for P2P energy trading, where there are important questions about sustainability, including how to support the participation and welfare of those in fuel poverty and how to support energy security 
through aggregating supply and demand [10]. In sum, in addition to understanding what motivates users, we also need to explore how these platforms can support social, economic and ecological sustainability.

\section{METHOD}

To explore how P2P energy trading is understood today by its prospective users, and how it could support sustainability, we undertook a series of in-depth interviews with people who have a self-defined interest in RE.

\section{Participants}

All participants $(n=45)$ were recruited by responding to advertisements placed in newsletters and social media channels of the host university as well as through community and cooperative energy groups in the UK. The advertisements asked for participants who have a self-defined interest in RE, however no prior knowledge of any P2P technology was required. We targeted individuals with interest in $\mathrm{RE}$ as we expect them to be one of the primary user groups of $\mathrm{P} 2 \mathrm{P}$ energy-trading platforms.

Most interviews (35) were conducted on a one-to-one basis. Yet, as we expect energy trading to be a household activity we also conducted 5 interviews with pairs of cohabiting partners. Our large interview sample aimed to meaningfully capture perspectives generators and consumers. Twenty-two of the forty households had solar photovoltaics, which is an ideal electricity generation technology for P2P energy markets. Participants were aged 29-86 years (median $=60$ years), and 14 identified as women (31 men).

\section{Design and Procedure}

Interviews were semi-structured and conducted either face-toface, over the phone or via videoconferencing software, they lasted approximately one hour. There was no compensation for participation, but travel costs were reimbursed. The interview consisted of two halves. The first half of the interview used open questions to explore participants' current interest in and involvement with RE (e.g., can you tell me about your current interest and involvement with RE?), perceptions of and experiences in the current UK energy market (e.g., can you tell me about your experiences in the current RE market?), and perceptions of a new P2P electricity market (e.g., can you explain to me what you think the benefits of a new P2P system would be?).

The second half of the interview used vignettes (e.g., [25, 36, $51,53]$ ) and sketches of possible designs (see Figure 1 for sample vignette and sketches), which functioned to probe how participants make decisions about key behaviors of interest. To begin with we wanted to explore how people make decisions about: (1) buying green energy vs fossil fuels; (2) using a P2P system vs traditional energy company; (3) maximizing profit vs contributing to fuel poverty; (4) trading as an individual vs trading as a group; and (5) automated trading. In turn, we wanted to explore what the prospective $\mathrm{P} 2 \mathrm{P}$ trading platform users would expect of such a platform.
Given that P2P markets can have a variety of configurations, our designs enabled different levels of localized trading, thirdparty regulation, and recommendation from authoritative sources. The sketches varied in terms of whether the trading platform was presented as: (1) a local vs national network; (2) recommended by the Government vs non-governmental advisory group vs other users; and (3) unregulated vs regulated by a national energy supplier. We created these designs by drawing on existing research examining P2P energy trading that emphasizes issues of location, trust and governance in these markets $[42,47,10]$.

To help the prospective users better relate to the P2P trading notions, we created a story about a character (Sali) who needed to make these decisions about their own energy, the character was either described as a buyer or seller of electricity. The story was made of 4-5 related scenarios, which mapped directly to our 5 behaviors of interest. Before we began the story participants were asked what they imagined their role in the new RE market to be (buyer, seller, or both), which determined the story they were told (we picked a story at random for participants who answered 'both'). Within each story we randomly varied the designs between participants. After each scenario participants were asked to describe: (1) risks and benefits of each option; (2) what the character should do and how they made the decision; and (3) what the interviewee themself would do and how they made the decision.

The interviews were recorded and transcribed verbatim, then analyzed using thematic analysis [9]. We adopted a contextualist approach to the analysis [9] and used a hybrid coding process, which develops themes and patterns from the data by combining deductive and inductive coding strategies [19]. To begin with, we arranged our analysis around two deductive categories: the ways that participants talked about DP and the ways they talked about a P2P electricity-trading platform. We chose these categories based on our primary research interests. We then followed the six steps of thematic analysis to develop five inductive themes.

\section{ANALYSIS AND P2P PLATFORM DESIGN IMPLICATIONS}

Here we consider two main questions:

1. How our participants talk about the decarbonization process of energy in general?

\section{How our participants talk about the P2P platform itself?}

\section{Decarbonization Process of Energy}

\section{A Component of a Sustainable Lifestyle}

The first theme described engagement with DP as a component of a sustainable lifestyle. Participants described consuming, generating and investing in RE as substantiable behaviors. In turn, participants characterize these behaviors as being bound up with other actions that they personally engage in to 'live sustainably'; for example, growing their own food or sourcing building materials locally.

Engagement with RE is characterized as supporting sustainability by: (1) doing something good for the planet; (2) using resources efficiently; and (3) increasing security. Specifically, 

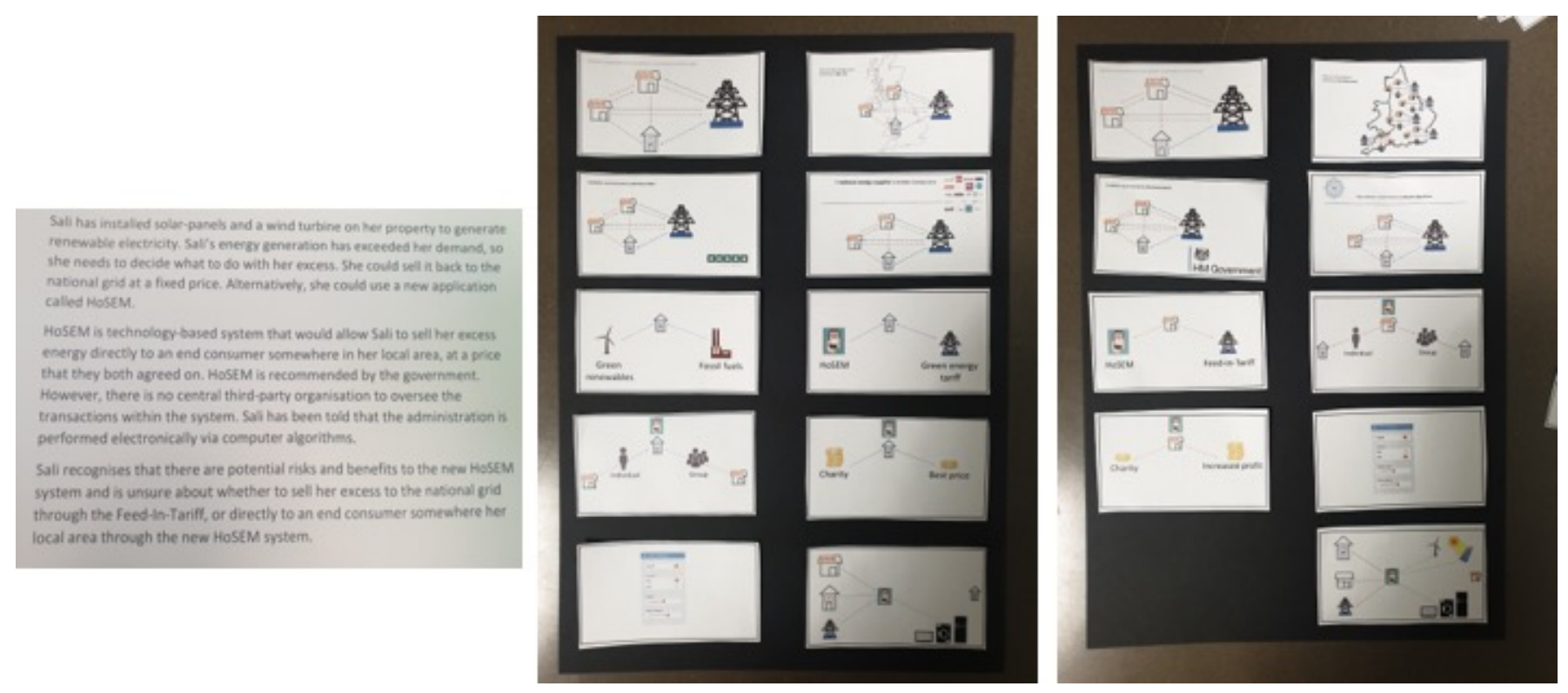

Figure 1. Sample vignette and sketch desings.

participants described the ecological benefits of renewables, which includes powering homes without contributing to climate change. They also described how RE prevents energy waste and supports a more effective use of limited resources. For example, not wasting the energy produced by the sun and freeing-up petrochemicals for pharmaceuticals. Similarly, they described how RE generation enables different types of security for different entities; includeing for individuals, communities and nations by enabling self-sufficiency and independence from centralized or foreign suppliers. In this way, participants described how their own personal engagement with DP is underpinned by different values, which center around different 'ways of being', including being 'green', resource-efficient, and secure. In turn, decarbonization initiatives are expected to support these values.

[It's] a good feeling that we're getting our heating... without fouling up the planet. (P1)

Design Implications for P2P platform: Given that the sustainable lifestyle appears to be a major driver for the interviewees, the impact that new energy-related activities (such as a $\mathrm{P} 2 \mathrm{P}$ trading) has on the environment, resource consumption, and energy security should be clearly visible to the platform users. This could be presented, for instance, in terms of amount of $\mathrm{CO} 2$ emissions saved through $\mathrm{P} 2 \mathrm{P}$ trading (for environmental impact); amount of oil/gas saved (for resource efficiency), and number of KWh of energy self-generated due to trading participation (for energy security).

\section{A Mechanism that Enables Individuals and Groups to Take} Ownership of Sustainability

The second way that participants talk about DP is as a mechanism that enables individuals and groups to take ownership of sustainability. Thus, decarbonization also represents a way of 'doing' - or organizing - society.
On the one hand, top-down governance structures - such as policy makers and energy supply companies - are characterized as occupying an ambivalent position: fulfilling important roles for DP, but also acting in their own interests and insensitive to the needs of UK society. For example, while participants welcomed Government subsidies for facilitating household generation, they were critical of subsidy reductions and skeptical of Government motives. Similarly, although alternative energy companies were characterized as vanguards of DP through souring their electricity entirely from renewables, 'the Big 6' energy companies were described entirely motivated by profit at the expense of customers and the environment.

[Privatized energy companies] will just carry on hiking and hiking prices to increase profits. No amount of profit is ever enough, they always want more. (P11)

In contrast, participatory modes of engagement were characterized as efficacious for DP; participation was frequently characterized as local in orientation, and individuals and membership groups were described as key participants in, and drivers and beneficiaries of, RE initiatives. For example, participants described how installing renewable generation on their houses allowed them to experience a sense of self-sufficiency and independence from private energy companies. Similarly, community and cooperative energy schemes, and workplace initiatives were characterized as a way to promote public participation, independence, social change. For example, participants described how - through local meetings and leadership opportunities - community and cooperative schemes create ways to engage where people are, raise community awareness, build community cohesion, and enable groups to have responsibility, decision-making power and ownership over the energy issues that affect them. They also outlined how these schemes bring tangible benefits for local communities through investment 
opportunities, keeping money in the local economy and steady streams of income.

In sum, generating and consuming RE are understood to be participatory modes of enacting decarbonization, in which individuals and membership groups are the primary leaders, actors and beneficiaries. In turn, these bottom-up modes of organization are described as mechanisms to facilitate community participation, independence and social change, thereby creating a virtuous cycle where participatory modes of enacting decarbonization support further benefits by encouraging the broader community to engage with DP.

At [cooperative energy scheme] there were a lot of face to face talks in village halls, and meetings and local community groups, to really get buy in at an individual level and then everybody understands actually more about their energy use, becomes more aware of it and then energy use changes. (P27)

Design Implications for P2P platform: The lack of trust in large energy companies and desire to localize the leadership and decision-making authority are likely to lead to dramatically differ models of P2P energy-related activities between various communities. This implies that the business models governing $\mathrm{P} 2 \mathrm{P}$ trading arrangements over the trading platform should be flexible. In other words, the platform should not provide a single model, but allow self-organization of platform users. If one community chooses to structure its trade activity into a cooperative for self-consumption optimization, and another chooses to organize into a for-profit energy trading entity, the platform should support both trading structures.

\section{Challenges in the Current System}

Our third theme described engagement with DP as challenging in the current system. Participants talked about challenges in three different ways.

Firstly, participants suggested that the broader public's 'hearts and minds' need to change to increase support for renewable generation technologies and participation in energy efficiency behaviors. Part of this discourse described how people have alternative priorities, which do not include decarbonization. Similarly, participants also talked about local objections to renewable generation technology; for example, how local RE schemes can face community opposition due to being 'too close'. Participants presented interesting discourses about knowledge and changing behavior; on the one hand, some characterized greater knowledge about energy and cognizant behavior change (e.g., shifting consumption to coordinate with solar generation) as integral to DP through leading to a trajectory of increased engagement. In contrast other participants presented a discourse that advocated for low-threshold (low effort/understanding) interventions that make engagement accessible by not requiring radical behavior change or deep understanding of energy (e.g., through energy-efficient lightbulbs, insulated homes).

Participants also talked about 'social structure'; they described policy makers, traditional energy supply companies (see Section 'A Mechanism that Enables Individuals and Groups to Take Ownership of Sustainability'), popular sources of infor- mation and financial measures as inadequate for increasing engagement with DP. For example, participants describe how it is difficult to obtain unbiased information about RE generation equipment as it primarily comes from commercial companies. Regarding financial dimensions, participants characterize RE tariffs and generation equipment as expensive and inaccessible. Similarly, they describe how the current billing system does not facilitate an understanding of energy or behavior change by emphasizing price rather than consumption. Thus, participants understood current resources as largely insufficient for mediating widespread change.

We only talk about money, so people only understand their bill... there's a complete disconnect between consumption and money. (P12)

Finally, participants described how limits to natural and technological resources create challenges for energy security and the environment. For example, how solar panels and batteries cannot support consumption over the winter due to reduced sunlight and high storage loss. Thus, even despite structural and psychology challenges, participants describe the need for technological advances to support DP. Specifically, a need to make RE constantly and readily available from sustainable sources.

Design Implications for P2P platform: To change the hearts and minds, as well as to circumvent the engagement hindered by lack of understanding of the hardware and financial and technical issues, the $\mathrm{P} 2 \mathrm{P}$ platform should (a) provide stagedengagement opportunities, and (b) serve as a continuous educational and information asset. The staged engagement can be supported by allowing participants with no self-generation to join the P2P trading platform, as consuming-only parties. Such participation would not require any initial financial or technology commitment but will provide the participants with an opportunity to benefit from the local generation, become part of the $\mathrm{P} 2 \mathrm{P}$ community, and learn. The learning could be delivered through informal chats between the platform participants about both hardware, beneficial practices, skill sharing, as well as formal statistical reports generated from the participation data, newsletters, and reports. As the participants gain more knowledge and confidence, they would likely wish to participate in the generation activities as well. Through the P2P platform, the community member could also set up partnerships with the local hardware installation companies, and other service providers, further helping with the engagement of their new and existing members.

\section{How do Participants Talk About a P2P Energy-Trading Platform?}

\section{A Tool that can Support Users in Shaping Society}

The first way that our participants talk about a P2P energytrading platform is as a tool that can support users in shaping society. Participants discuss the platform in terms of its capacity to enable them to address the challenges in the current system, support DP and broader sustainability goals. Participants outline two different ways that the platform could support this type of social change. 
Empowering Individuals and Community Groups to Meet the UK's Energy Needs

First, they depicted the platform as a resource that could enable a network of small, distributed suppliers to meet the UK's energy management needs. Part of this discourse described how novel technical and economic features might of the platform might automatically enable better electricity prices, and a more efficient electricity network. However, participants go further than this and depict this process as changing the behavior of users and non-users alike. For example, they described how it could increase the supply of RE by supporting new and better mechanisms for return-on-investment beyond government subsidies, thereby stimulating investment in local generation schemes. Similarly, participants described how local schemes could increase community support for, and engagement with, local schemes by supporting benefits for the local community, such as less expensive electricity supply.

Similarly, participants talk about the system's capacity (both positive and negative) to shape energy demand by enabling users to have different levels of awareness of - and engagement with - energy. On the one hand, participants present a discourse that describes how an automated trading process could reduce awareness and understanding of energy by disconnecting energy from people's lives. However, they also describe the opposite situation where participation in a $P 2 P$ trading platform would increase the commitment that most people have to energy as a concept, and therefore reduce consumption.

Thus, participants described how P2P energy markets could facilitate engagement in DP by supporting different types of psychological (i.e. hearts and minds) and structural (i.e. economic, technical) change, which enable users to have a greater sense of control over energy supply and demand. Moreover, they described how these technologies could support psychological and financial investment in renewables by those who are not users of the platform, through supporting engagement and shared benefit between users and other groups (e.g., investors, local community).

Wouldn't it be better if communities were independently powered and looked after their own power sources... [with technology] it can be done in a much more scientific technical way. (P1)

\section{Creating Opportunities for New Social Relationships}

The second subtheme described the platform as supporting users to shape the social relations that are central to DP. The ability to trade between individuals and groups is characterized as creating opportunities for different kinds of social relationships. At the community level this involves supporting greater social connections and cohesion within local communities, by "build[ing] up a community where people want to feel like they're committing to the community" (P18). Thus, it is described as a mechanism for facilitating community responsibility and relations. At the system level, participants talk about the platform as having the capacity to support changing user relationships with policy makers and energy supply companies. Specifically, by enabling users to make decisions about their energy supply and excess generation as an alterna- tive to top-down and centralized control over the UK's energy challenges.

People having control ... they're generating energy and they can profit from that and they can do that how they see fit. (P18)

Thus, in this theme we see that rather that only supporting personal economic benefit, $\mathrm{P} 2 \mathrm{P}$ energy markets are valued when they support users in changing the relationships and culture that shape DP, by enabling users to have: (1) a different type of engagement with energy through supporting greater awareness and understanding; and (2) different relationships with others through supporting community cohesion, decisionmaking power, and benefit.

Nevertheless, our participants considered these benefits to be neither automatic nor inherent. In our final theme participants presented a discourse of 'benefit for whom', which described the benefits of $\mathrm{P} 2 \mathrm{P}$ energy trading as being contingent on the way that platforms are designed.

Design Implications for P2P platform: Here the social and cultural change is driven by individuals who organize into like-minded groups and share their views with newcomers. To support the groups in expressing their views and enabling other like-minded individuals to join these groups, the $\mathrm{P} 2 \mathrm{P}$ platform should enable explicit verbalization of the group's goals and objectives (e.g., by providing a description of these for each group on the platform). For instance, if a group is formed with a goal to alleviate fuel poverty in the Easton community of Bristol city, it could have a policy that each member will donate $5 \%$ of their excess generation to the Easton's fuel poverty fund, which will then be distributed to those in Easton who are identified as fuel poor (e.g., through city council's register) free of charge. This implies that anyone joining the given group volunteers the specified portion of their generation for the specified cause. Such features within the platform also imply that while some groups will be focused around geographical localities, others may be centered on ideological grounds (e.g., animal rights groups, etc.). Thus, the platform should support geographically co-located as well as widely distributed community trades. It should be noted that such solutions are likely to have different implications for the physical power systems over which the energy trading is taking place: while geographically localized groups may lead to localization of the physical infrastructure, the distributed groups will continue to rely on the interconnected national (and international) energy grid.

\section{Responses to the Platform Itself}

Our final theme is concerned with participants' responses to the platform itself. Different elements are talked about in terms of having the potential to encourage and/or hinder platform use and support different types of benefit.

\section{Increased Digitization, Increased Risk}

First, participants describe how P2P energy-trading platforms would mean an increasingly digitized energy market. They draw on personal experience and social narratives about digital technology to describe how increased digitization presents increased risk. 
One concern is transparency: participants want to be able to access and understand information about how decisions are made about payments and energy supply. For example, they described a negative situation where users are unable to understand "why you're getting what you're getting" (P2). They use discourses of simplicity, visibility and honesty to describe desired values.

Participants also express concerns about privacy: that their data is secure and confidential. For example, one participant talked about the risks of "advertising the fact to the world that I'm on holiday" (P25) because they're not consuming electricity at home. Perhaps due to the P2P nature of the system, participants were concerned that sensitive information could be directly exposed or inferred by other users. A further concern was hacking, both in terms of people exploiting weaknesses in the system to gain access to other digital tools like bank accounts, and users manipulating the system itself to misrepresent their own generation or consumption. Similarly, participants described how a new or untested system might be unable to offer the same customer protections as the traditional energy market, in terms of secure supply and accurate billing. Thus, P2P energy markets were understood to be potentially less secure than the traditional energy market. Nevertheless, participants described how trust could be facilitated by knowing who you are trading with, where energy has come from, who is benefiting from production, and that production is sustainable. Given the importance of privacy, participants outlined how knowing "the population as without knowing specific individuals ... who's using in general terms, in terms of the shape of the community" (P2) would facilitate good feelings, participation and a sense of community without being intrusive.

Finally, participants talked about digital exclusion and age. They highlighted that, in the current market, older friends and relatives were financially disadvantaged because limited internet access, low computer literacy, and a distrust of digital technology left them unable to access online-centric benefits, such as switching suppliers or discounts for paperless billing. They expressed concerns that a market that was only accessible via digital technology would further exacerbate these issues. In contrast, participants described the benefits of being able to "talk to an individual about it and talk through some of [your] concerns and worries"(P18). At the same time, they described how mechanisms for 'offline' trading could be supported through the platform. For example, a communityowned battery that was housed in a community center with solar panels where members of the community could come and recharge their own batteries from this community source.

If a community center, which might already have panels, had really quite a big battery and there was a community ownership scheme for batteries, which also permitted sale on a peer-to-peer basis. So if you own a slice of the battery and it was charged and discharged during the day or whatever, with obviously a difference in the price, you could decide who you sold it to. (P23)

\section{Different Power Relations, Same Lack of Power}

Our second subtheme described power relations: putting power in the hands of other people or algorithms. Drawing on some of the aforementioned discourses about digitization, participants characterized $\mathrm{P} 2 \mathrm{P}$ energy markets as enabling new and different types of power relations, but potentially the same position of low power for everyday users.

There was a discourse about automation. Participants described how algorithms make it difficult to understand "who's monitoring you, how's it been designed, what's it looking for and how is it going to work out" (P22). However, automation could also be seen as potentially empowering, if participants could indicate their preferences: they described how users could set up the system and then carry on with their lives without having to exert further effort or concern.

Similarly, participants also present a discourse about big tech corporations having control in the place of large energy companies and at the expense of users. Participants draw on narratives such as the Facebook-Cambridge Analytical data scandal to characterize how technology owners could exploit the lack of transparency and personal information within the system for their own benefit.

Likewise, they describe concerns about unequal relationships between users. For example, one participant described the platform enabling sellers have power over buyers by deciding who to sell to at what cost. They expressed concerns that consumers could be judged by suppliers and not sold to, or sold to at a higher cost. There were similar concerns that some users could be disadvantaged in price negotiations if the tool were individualized in nature with limited safeguards.

Making the individual [negotiate]... I feel I could personally handle a situation like that because... I'm fairly streetwise... [my son] is a solicitor... but an awful lot of people haven't got those safeguards. (P1)

Thus, our participants describe how P2P energy markets can complicate notions of control and present challenges for empowerment. In turn, in our final subthemes, participants described how the structural organization of a P2P energy network might enhance or diminish these concerns.

\section{Large Networks for Trusted Energy Supply, Small Networks for Trusting People}

The first way that participants talk about structure is in terms of the size and geographical boundaries of a P2P energy market. They describe a tension between larger networks that offer higher levels of energy security, and small local networks that enable users to feel a sense of responsibility over energy and other local issues, and perhaps the feeling of security about personal data. For example, how a small local network would allow community members to take responsibility about what they're producing and using, and how it might be less open for abuse. In contrast, a larger network is described as being better equipped to deal with fluctuations in supply and demand, and perhaps economically fairer in locations with limited generation capacity by increasing supply. In addition to grouping by geography, participants describe how aggregation of supply 
and demand could be facilitated by enabling users to come together in small groups made up of like-minded users with elected group leaders. Participants describe how coalescing with like-minded others would increase trust between users, and willingness to defer decision-making to elected leaders.

I think it could happen on two levels. it could be an international global peer thing ... Or it could be local, I am much more interested in doing whatever is possible at a local level. Because with energy there are different issues that you can solve locally. (P27)

\section{Participation and Decentralization, not Structurelessness}

In our final subtheme participants discuss the need for the system to enable a third-party - beyond the users and technology developers - to govern and regulate the system. Considering the aim for individual and group participation in DP, they describe: (1) the different types of governance that could be appropriate; and (2) who should occupy a governing role. Rather than coming to any concrete conclusions, participants drew on notions of independence, ethics, transparency and user benefit to describe different entities as more or less desirable. Moreover, participants described how an official form of governance could provide added benefits over mere 'recommendation' from trustworthy sources.

Participants describe a variety of governance mechanisms including new regulations, charters, and organizations that oversee the tool and transactions. Part of this included a discourse about energy, financial and data security for users. However, participants also described how governance structures could support those in fuel poverty. To begin with, they described how a P2P market might leave low-income households at the mercy of other users' altruism and charitable giving, which stands in contrast to the current market where protections for fuel poverty are built-in. Although some participants suggest that charitable donations could be increased by providing assurances about where and how donations are being spent, others suggested that a third-party such as national Government should be responsible for providing support.

In terms of who should occupy a governing role more generally, several suggestions were provided including charities, non-profits, entrepreneurs, technology companies, local councils and cooperative energy schemes. They described how any form of governance should uphold certain values including ethical and transparent conduct. Moreover, they emphasized how commercial companies might need additional regulation to be effective in this context.

Somebody's got to run it, but I'm not really sure whether I think it should be a registered charity... [a company running it for profit would need] very clear regulations and, I don't know, a charter, to know what they're doing and why, and very clear limitations about what can be changed in the future. (P6)

Design Implications for P2P platform: The ability to ensure participants' privacy and security is central to the willingness of the prospective users to engage with the trading platform. In particular, issues of data ownership, access to data, and data monetization have to be resolved, ensuring that the
P2P platform participants (who are also the data subjects) have full ownership and secure control over it. The precise governance structure would significantly depend on the specific business model agreed upon by each community (as discussed in section 'A Mechanism that Enables Individuals and Groups to Take Ownership of Sustainability' above).

To ensure that each small network of trusted people (i.e., a $\mathrm{P} 2 \mathrm{P}$ trading group/community) is assured access to larger than itself energy trading network, the P2P platform must provide wider linked-up infrastructure either to the national grid, or to many other P2P networks. One could envision either a "supplier of last resort" contract between the P2P community and the grid, whereby the community purchases a kind of an insurance service from the gird that energy demand would be satisfied, should such demand arise at any given time. Alternatively, similar contracts or mutual support could be established between several P2P communities that would ideally harness renewable energy from a mix of sources, providing back-up supply when one of the renewable sources (e.g., sunlight for PV generation at night time) falls short. Additionally, integration of long-term storage facilities (e.g., hydro stations, bio-gas banks, etc.) into the P2P platform network would also help to alleviate the supply security concerns. Finally, to enable engagement of less digitally abled, the P2P trading platform that aims for just transition to a clean energy system would provide energy trading participation as a service, whereby such users could subscribe to the P2P trading service as they currently subscribe to an energy consumption service with their energy utilities.

\section{DISCUSSION}

Our analysis provides evidence of the ways that prospective users of P2P energy markets understand and seek to engage in $\mathrm{P} 2 \mathrm{P}$ modes of energy trading. We demonstrate how P2P energy trading is valued as a mechanism to advance social and ecological goals. In particular, we demonstrate the values of collective and community-level energy trading for facilitating sustainable lifestyles, building meaningful interactions within communities, and enabling communities to become empowered agents in the energy market. We also presented a series of design characteristics, outlining how P2P energy-trading platforms can support users in: (1) understanding the impact of P2P trading on the environment; (2) accessing flexible business models; (3) staged engagement and continuous education; (4) the externalization of group goals and objectives; and (5) engaging with bridging infrastructures. In turn, we suggest that these recommendations provide a way to meet participants' expectations and concerns about P2P energy trading. In the following subsections we discuss our analysis and design implications in relation to our main research question and behaviors of interest.

\section{Choosing Green Energy and a P2P System}

To begin with, we found that participants valued the ways in which P2P energy markets can support widespread engagement with energy issues. Given the value of sustainable lifestyes, in order to support participation in P2P energy trading we suggest finding ways to support users in understanding the impact of $P 2 P$ trading on the environment. 
Our analysis also highlights the value of changed intergroup relationships. Part of this involves enabling prospective users as a group to become empowered through greater decisionmaking. At the same time, our findings highlight the value of tangible benefits for local communities and groups; in particular, how locally-oriented trading provides opportunities to increase community support for local schemes by enabling direct benefits to local communities. Thus, participants valued the ways that P2P energy markets could support users in transferring the responsibility for - and benefits of - energy generation and management from private companies to local citizens. Accordingly, we advocate for flexible business models that enable users to self-organize and structure trade activity to meet self-determined goals.

It should be noted that the need or the variety of such business models has already started to emerge in business practice, e.g, the Brooklyn trial focused on trading directly between peers [43], the Btixoton trial [2] looks at shared self-consumption, energy donation to community and exchange, while the Sonnen model [3] provides free energy use for assured company access to a portion of the household's battery storage. Despite the variety of the ways in which the P2P energy trading is carried out, all these activities have a common core platform functionality, and could be provided through a single platform, if it supports flexible self-organisation of participants into different buisness models.

\section{Interactions Within and Between Groups}

We found that participants valued the intragroup dimensions P2P energy markets. Participants understood these platforms as providing opportunities to build relationships within communities. Part of this was bound-up with expectations about the geographical boundaries and structures of these networks: participants understood $\mathrm{P} 2 \mathrm{P}$ trading platforms as supporting local and community-led trading. At the same time, there was the expectation of 'like-mindedness' between users, which presented opportunities to coalesce and trade electricity around shared ideologies. Given the value of shared group membership, our analysis indicates that $\mathrm{P} 2 \mathrm{P}$ energy markets can provide opportunities to organize around like-minded groups by enabling users to externalize goals and values. However, our analysis also suggests that P2P energy markets need to enable wider linked-up infrastructure that supports interactions between communities: participants highlighted a tension between the value of small trusted groups and the desire for larger networks for assurance of energy supply. Thus, these $\mathrm{P} 2 \mathrm{P}$ energy-trading platforms need to provide opportunities to balance these concerns.

Although prior research has examined ways to facilitate a technical understanding of $\mathrm{P} 2 \mathrm{P}$ energy trading and multifaceted stakeholder relationships [42, 47], our analysis builds on this work by demonstrating the ways that participants valued the social dimensions of P2P energy trading, and saw opportunities to build and cultivate social and collective resources through platform use. We have suggested that the design of P2P energy markets consider the value of enhanced intragroup relationships by enabling users to externalize values and organize around shared group memberships. At the same time,

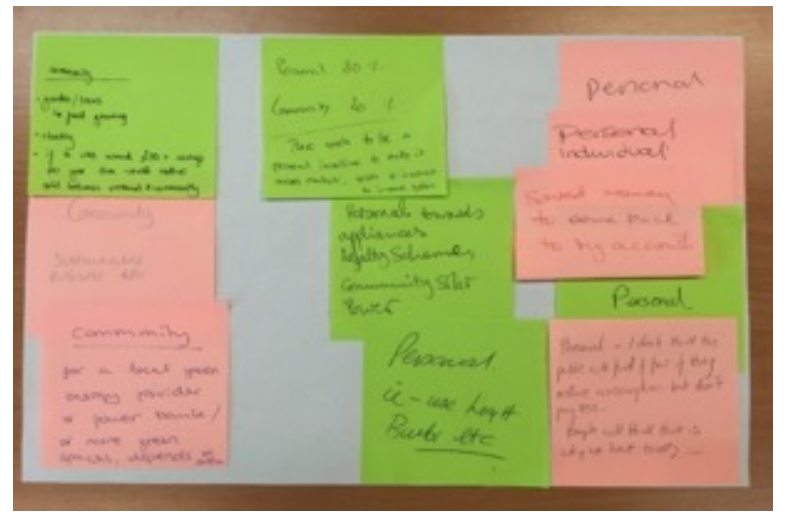

Figure 2. Use of financial savings from energy saving activities: personal vs communal goals.

our analysis emphasizes the importance of providing the infrastructure to interact between groups. Although existing research has examined how sharing-economy applications can support social experiences $[8,28,29]$, it has tended to focus on supporting relationships between individuals. In contrast, we highlight opportunities for supporting users to plug-in to and harness meaningful groups.

Our interview analysis suggested that individuals in a group would likely choose to use financial benefits obtained from energy/cost saving activities for a common goal. To validate this suggestion we asked a group of 12 low income householders, who were knowledgeable about RE, to anonymously write down if they would like to keep funds from energy saving activities to themselves or use them for some common purpose. This question was asked as part of a co-design workshop for a demand-side reposes service provision, which was not directly related to P2P trading, and had participants enrolled through city hall records. The participants were requested to leave anonymous opinions on a sticky notes (on an unmonitored wall) on their way out. These responses are shown in Figure 2. Out of the 11 responses received 6 wanted to use funds for themselves, 3 opted for fully communal use, and 2 wanted to share the savings between personal and community use. Though this small exercise does not provide any generalizable conclusion, it does validate the desire for common good and common goals voiced by the present interview study participants.

\section{Fuel Poverty and Automated trading}

Although distinct concerns, participants understood issues around fuel poverty and automation in terms of structurelessness. Automated trading, that takes account of user preferences, was understood to be a mechanism to support widespread engagement with DP by offering low-threshold entry to participation. However, automation had negative implications for some: it has the potential to conceal energy consumption, and could exacerbate digital exclusion. Similarly, while some participants valued the opportunity to make donations towards fuel poverty within the energy market, others argued for more formal support mechanisms. We have suggested that structures are put in place to support vulnerable 
populations in participating and benefiting from $\mathrm{P} 2 \mathrm{P}$ energy markets; this includes proving opportunities for staged engagement and continuous education, energy trading as a service, and group-led policies for charitable donations.

Existing research emphasizes support for digital literacy, simple yet transparent pricing structures, brand visibility and infrastructures that support financial inclusion in order to enable empowerment and trust [15, 31]. For example, installing public kiosks where people can access ridesharing services and participate in training about transactions [15]. Our analysis also suggests that participants valued support for greater inclusion in - and understanding of - P2P energy markets. However, our findings extend this work by emphasizing the value of structures that help regulate the market and support users in making decisions about how these markets are run. We argue that the design of P2P energy markets should take account of these values to enable the empowerment of users as a group. Although previous research examining the sharing economy highlights the benefits and challenges of evidence-based and top-down policy $[11,48]$, we outline how structuring opportunities can be provided within-app and decided on by users themselves.

\section{Limitations and Future Work}

In the present study we were limited to a specific context, which is the UK energy market, and we also engaged with a specific set of participants who had a pre-existing interest in renewable energy. Thus, we can't be sure of the extent to which our findings generalize to other national contexts and populations. For example, individuals who have a primary interest in technology or business opportunities might have different needs and wants, understand P2P energy trading in different ways, and value other aspects of these markets.

We also highlight that our research was speculative, participants had not used a P2P energy-trading platform before, and therefore could only reflect on their own imaginations and perceptions of participation. This was partly due to current regulatory barriers in the UK that prohibit $\mathrm{P} 2 \mathrm{P}$ energy trading between microgenerators, which meant that we could not deploy a functional P2P energy-trading platform nor speak to individuals who had previously used these tools. Similarly, our study only analyzed talk, we were unable to observe behavior or test participants' lay hypotheses around the factors that would support participation and sustainability, nor do we claim to do this. Rather we were primarily interested in the ways that participants understood these phenomena.

Finally, our analysis examined P2P energy-trading platforms in general, today specific technologies, such as distributed ledger technologies (DLT), are a large part of the narrative around P2P energy markets [13, 27, 50, 54], however this study is technology agnostic.

As well as addressing these limitations, future research should examine how communities with low resources and limited capacity for renewable generation understand and seek to participate in $\mathrm{P} 2 \mathrm{P}$ energy markets. Evidence suggests that decarbonization in general, and some P2P energy trading models in particular, could place additional burdens on low-resource households and communities [10,46]. Thus, it is important to explore implications for these populations. Our research also highlighted how automated trading might influence engagement with DP more generally. Future research could examine when and how automated energy trading affects widespread and deeper engagement with energy. This would present opportunities to explore the intersection between humans, P2P energy markets, Internet of Things devices and DLT, and how these entities could work together in partnership to selectively trade electricity to achieve sustainability goals (e.g., [30]).

\section{Conclusion}

Our work extends HCI research examining P2P energy markets by presenting an analysis of how prospective users understand, value and seek to engage in these platforms, with a focus on members of the public who have a pre-existing interest in renewable energy. Our participants valued experiences that enable communities to organize around shared values, build an understanding of $\mathrm{P} 2 \mathrm{P}$ platforms and the ways that trading impacts the environment, and to configure their own selective engagement with business models and broader energy infrastructure. Our analysis emphasizes the importance of nurturing and supporting groups within P2P energy-trading platforms, particularly for populations who already have an interest and engagement with renewable energy. Likewise, our findings are consistent with existing research that highlights the need for autonomy, control and economic equality in these markets. More broadly, our research speaks to examinations of the sharing economy, we bring together research that emphasizes the social dimensions of sharing with calls to examine the social justice implications of sharing-economy applications. Specifically, we outline how users can harness groups to advance sustainability aims and how platforms can provide infrastructure and opportunities to support these efforts. Thus, our contributions can provide insights for the HCI community, private companies and the policy makers in developing P2P energy markets.

\section{ACKNOWLEDGMENTS}

This research is supported by the Anonymous Grant. Authors also gratefully acknowledge Anonymous Colleague for insightful discussions on P2P energy markets.

\section{REFERENCES}

[1] 2016. Clean Energy for All Europeans. Technical Report. Eurpopean Commission. 1-13 pages.

https://eur-lex. europa. eu/resource.html?uri=cellar: fa6ea15b-b7b0-11e6-9e3c-01aa75ed71a1.0001.02/DOC_1\& format=PDF

[2] 2019. EDF-backed solar, storage and blockchain pilot to get underway. (Feb. 2019).

https://www . current-news. co.uk/news/

edf-backed-solar-storage-and-blockchain-pilot-to-get-underway

[3] 2019. Sonnen Community. (2019). https://sonnenbatterie.co.uk/sonnencommunity/

[4] Merlinda Andoni, Valentin Robu, David Flynn, Simone Abram, Dale Geach, David Jenkins, Peter McCallum, and Andrew Peacock. 2019. Blockchain technology in 
the energy sector: A systematic review of challenges and opportunities. Renewable and Sustainable Energy

Reviews 100 (Feb. 2019), 143-174. DOI:

http://dx.doi.org/10.1016/j.rser.2018.10.014

[5] Kelvin Anoh, Bamidele Adebisi, Olamide Jogunola, and Mohammad Hammoudeh. 2017. Cooperative Hybrid Wireless-Powerline Channel Transmission for Peer-to-Peer Energy Trading and Sharing System. In Proceedings of the International Conference on Future Networks and Distributed Systems (ICFNDS '17). ACM, New York, NY, USA. DOI :

http://dx.doi.org/10.1145/3102304.3102311 event-place: Cambridge, United Kingdom.

[6] Kelvin Anoh, Augustine Ikpehai, Dragana Bajovic, Olamide Jogunola, Bamidele Adebisi, Dejan Vukobratovic, and Mohammad Hammoudeh. 2018. Virtual Microgrids: A Management Concept for Peer-to-peer Energy Trading. In Proceedings of the $2 \mathrm{Nd}$ International Conference on Future Networks and Distributed Systems (ICFNDS '18). ACM, New York, NY, USA, 43:1-43:5. DOI :

http://dx.doi.org/10.1145/3231053.3231096 event-place: Amman, Jordan.

[7] Victoria Bellotti, Alexander Ambard, Daniel Turner, Christina Gossmann, Kamila Demkova, and John M. Carroll. 2015. A Muddle of Models of Motivation for Using Peer-to-Peer Economy Systems. In Proceedings of the 33rd Annual ACM Conference on Human Factors in Computing Systems (CHI'15). ACM, New York, NY, USA, 1085-1094. DOI :

http://dx.doi.org/10.1145/2702123.2702272

[8] Victoria M.E. Bellotti, Sara Cambridge, Karen Hoy, Patrick C. Shih, Lisa Renery Handalian, Kyungsik Han, and John M. Carroll. 2014. Towards Community-centered Support for Peer-to-peer Service Exchange: Rethinking the Timebanking Metaphor. In Proceedings of the SIGCHI Conference on Human Factors in Computing Systems (CHI'14). ACM, New York, NY, USA, 2975-2984. DOI :

http://dx.doi.org/10.1145/2556288.2557061 event-place: Toronto, Ontario, Canada.

[9] Virginia Braun and Victoria Clarke. 2006. Using thematic analysis in psychology. Qualitative Research in Psychology 3, 2 (Jan. 2006), 77-101. DOI : http://dx.doi.org/10.1191/1478088706qp063oa

[10] R. Bray, B. Woodman, and P. Connor. 2018. Policy and regulatory barriers to local energy markets in Great Britain. Working Paper. University of Exeter, Energy Policy Group. https:

//ore.exeter.ac.uk/repository/handle/10871/33607

[11] Sally Caird, Robin Roy, and Horace Herring. 2008. Improving the energy performance of UK households: Results from surveys of consumer adoption and use of low- and zero-carbon technologies. Energy Efficiency 1, 2 (June 2008), 149. DOI :

http://dx.doi.org/10.1007/s12053-008-9013-y
[12] Sid Chi-Kin Chau, Jiajia Xu, Wilson Bow, and Khaled Elbassioni. 2019. Peer-to-Peer Energy Sharing: Effective Cost-Sharing Mechanisms and Social Efficiency. ACM, 215-225. DOI :

http://dx.doi.org/10.1145/3307772.3328312

[13] Ruzanna Chitchyan and Jordan Murkin. 2018. Review of Blockchain Technology and its Expectations: Case of the Energy Sector. arXiv:1803.03567 [cs] (March 2018). http://arxiv.org/abs/1803.03567 arXiv: 1803.03567.

[14] Tawanna Dillahunt, Vaishnav Kameswaran, Linfeng Li, and Tanya Rosenblat. 2017. Uncovering the Values and Constraints of Real-time Ridesharing for Low-resource Populations. In Proceedings of the 2017 CHI Conference on Human Factors in Computing Systems (CHI '17). ACM, New York, NY, USA, 2757-2769. DOI : http://dx.doi .org/10.1145/3025453.3025470 event-place: Denver, Colorado, USA.

[15] Tawanna Dillahunt, Airi Lampinen, Jacki O'Neill, Loren Terveen, and Cory Kendrick. 2016. Does the Sharing Economy Do Any Good?. In Proceedings of the 19th ACM Conference on Computer Supported Cooperative Work and Social Computing Companion (CSCW'16 Companion). ACM, New York, NY, USA, 197-200. DOI : http://dx.doi .org/10.1145/2818052 .2893362 event-place: San Francisco, California, USA.

[16] Geanderson E. dos Santos, Pedro H. F. Holanda, Jussara M. Almeida, and Raquel O. Prates. 2017. Characterizing Quality Aspects in Airbnb. In Proceedings of the XVI Brazilian Symposium on Human Factors in Computing Systems (IHC 2017). ACM, New York, NY, USA, 56:1-56:4. DOI :

http://dx.doi.org/10.1145/3160504.3160571 event-place: Joinville, Brazil.

[17] Cherrelle Eid, Paul Codani, Yannick Perez, Javier Reneses, and Rudi Hakvoort. 2016. Managing electric flexibility from Distributed Energy Resources: A review of incentives for market design. Renewable and Sustainable Energy Reviews 64 (Oct. 2016), 237-247. DOI : http://dx.doi.org/10.1016/j.rser.2016.06.008

[18] Anton Fedosov, Airi Lampinen, Tawanna R. Dillahunt, Ann Light, and Coye Cheshire. 2019. Cooperativism and Human-Computer Interaction. In Extended Abstracts of the 2019 CHI Conference on Human Factors in Computing Systems (CHI EA '19). ACM, New York, NY, USA, SIG05:1-SIG05:4. D0I :

http://dx.doi.org/10.1145/3290607.3311751 event-place: Glasgow, Scotland Uk.

[19] Jennifer Fereday and Eimear Muir-Cochrane. 2006. Demonstrating Rigor Using Thematic Analysis: A Hybrid Approach of Inductive and Deductive Coding and Theme Development. International Journal of Qualitative Methods 5, 1 (March 2006), 80-92. DOI : http://dx. doi .org/10.1177/160940690600500107 
[20] Derek Foster, Shaun Lawson, Mark Blythe, and Paul Cairns. 2010. Wattsup?: Motivating Reductions in Domestic Energy Consumption Using Social Networks. In Proceedings of the 6th Nordic Conference on Human-Computer Interaction: Extending Boundaries (NordiCHI '10). ACM, New York, NY, USA, 178-187. DOI : http://dx.doi .org/10.1145/1868914.1868938 event-place: Reykjavik, Iceland.

[21] Derek Foster, Shaun Lawson, Jamie Wardman, Mark Blythe, and Conor Linehan. 2012. "Watts in It for Me?": Design Implications for Implementing Effective Energy Interventions in Organisations. In Proceedings of the SIGCHI Conference on Human Factors in Computing Systems (CHI'12). ACM, New York, NY, USA, 2357-2366. DOI :

http://dx.doi.org/10.1145/2207676.2208396 event-place: Austin, Texas, USA.

[22] Mareike GlÃúss, Moira McGregor, and Barry Brown. 2016. Designing for Labour: Uber and the On-Demand Mobile Workforce. In Proceedings of the 2016 CHI Conference on Human Factors in Computing Systems (CHI '16). ACM, New York, NY, USA, 1632-1643. DOI : http://dx.doi.org/10.1145/2858036.2858476

[23] Yukang Guo, Matt Jones, Benjamin Cowan, and Russell Beale. 2013. Take It Personally: Personal Accountability and Energy Consumption in Domestic Households. In CHI'13 Extended Abstracts on Human Factors in Computing Systems (CHI EA '13). ACM, New York, NY, USA, 1467-1472. DOI :

http://dx.doi.org/10.1145/2468356.2468618 event-place: Paris, France.

[24] Florian Heller, Konstantinos Tsoleridis, and Jan Borchers. 2013. Counter Entropy: Visualizing Power Consumption in an Energy+ House. In $\mathrm{CHI}$ '13 Extended Abstracts on Human Factors in Computing Systems (CHI EA '13). ACM, New York, NY, USA, 2363-2366. DOI :

http://dx.doi.org/10.1145/2468356.2468771 event-place: Paris, France.

[25] Rhidian Hughes and Meg Huby. 2012. The construction and interpretation of vignettes in social research. Social Work and Social Sciences Review 11, 1 (Dec. 2012), 36-51. DOI : http://dx. doi .org/10.1921/swssr.v11i1.428

[26] Tapio Ikkala and Airi Lampinen. 2015. Monetizing Network Hospitality: Hospitality and Sociability in the Context of Airbnb. In Proceedings of the 18th ACM Conference on Computer Supported Cooperative Work \& Social Computing (CSCW'15). ACM, New York, NY, USA, 1033-1044. DOI :

http://dx.doi.org/10.1145/2675133.2675274

event-place: Vancouver, BC, Canada.

[27] F. Imbault, M. Swiatek, R. de Beaufort, and R. Plana. 2017. The green blockchain: Managing decentralized energy production and consumption. In 2017 IEEE International Conference on Environment and Electrical
Engineering and 2017 IEEE Industrial and Commercial Power Systems Europe (EEEIC / I CPS Europe). 1-5. DOI : http://dx.doi.org/10.1109/EEEIC.2017.7977613

[28] Jiwon Jung, Susik Yoon, SeungHyun Kim, SangKeun Park, Kun-Pyo Lee, and Uichin Lee. 2016. Social or Financial Goals?: Comparative Analysis of User Behaviors in Couchsurfing and Airbnb. In Proceedings of the 2016 CHI Conference Extended Abstracts on Human Factors in Computing Systems (CHI EA '16). ACM, New York, NY, USA, 2857-2863. DOI : http://dx.doi.org/10.1145/2851581.2892328

[29] Vaishnav Kameswaran, Lindsey Cameron, and Tawanna R. Dillahunt. 2018. Support for Social and Cultural Capital Development in Real-time Ridesharing Services. In Proceedings of the 2018 CHI Conference on Human Factors in Computing Systems (CHI '18). ACM, New York, NY, USA, 342:1-342:12. DOI: http://dx.doi.org/10.1145/3173574.3173916 event-place: Montreal QC, Canada.

[30] J. Kang, R. Yu, X. Huang, S. Maharjan, Y. Zhang, and E. Hossain. 2017. Enabling Localized Peer-to-Peer Electricity Trading Among Plug-in Hybrid Electric Vehicles Using Consortium Blockchains. IEEE Transactions on Industrial Informatics 13, 6 (Dec. 2017), 3154-3164. DOI : http://dx.doi.org/10.1109/TII.2017.2709784

[31] Qing Ke. 2017. Service Providers of the Sharing Economy: Who Joins and Who Benefits? Proc. ACM Hum.-Comput. Interact. 1, CSCW (Dec. 2017), 57:1-57:17. DOI : http://dx.doi .org/10.1145/3134692

[32] Ju-Whan Kim, Yun-Kyung Kim, and Tek-Jin Nam. 2009. The TÃl'NÃl'RÃl': Design for Supporting Energy Conservation Behaviors. In CHI '09 Extended Abstracts on Human Factors in Computing Systems (CHI EA '09). ACM, New York, NY, USA, 2643-2646. DOI : http://dx.doi.org/10.1145/1520340.1520372 event-place: Boston, MA, USA.

[33] Jesper Kjeldskov, Mikael B. Skov, Jeni Paay, Dennis Lund, Tue Madsen, and Michael Nielsen. 2015.

Facilitating Flexible Electricity Use in the Home with Eco-Feedback and Eco-Forecasting. In Proceedings of the Annual Meeting of the Australian Special Interest Group for Computer Human Interaction (OzCHI '15). ACM, New York, NY, USA, 388-396. DOI : http://dx.doi.org/10.1145/2838739.2838755 event-place: Parkville, VIC, Australia.

[34] Airi Lampinen, Victoria Bellotti, Andres Monroy-Hernandez, Coye Cheshire, and Alexandra Samuel. 2015. Studying the "Sharing Economy": Perspectives to Peer-to-Peer Exchange. In Proceedings of the 18th ACM Conference Companion on Computer Supported Cooperative Work \& Social Computing (CSCW'15 Companion). ACM, New York, NY, USA, 117-121. DOI :

http://dx.doi.org/10.1145/2685553.2699339 event-place: Vancouver, BC, Canada. 
[35] Airi Lampinen and Coye Cheshire. 2016. Hosting via Airbnb: Motivations and Financial Assurances in Monetized Network Hospitality. In Proceedings of the 2016 CHI Conference on Human Factors in Computing Systems (CHI'16). ACM, New York, NY, USA, 1669-1680. DOI :

http://dx.doi.org/10.1145/2858036.2858092

[36] Robert Loo. 2002. Tackling ethical dilemmas in project management using vignettes. International Journal of Project Management 20, 7 (Oct. 2002), 489-495. DOI : http://dx.doi .org/10.1016/S0263-7863(01)00056-4

[37] Xing Luo, Jihong Wang, Mark Dooner, and Jonathan Clarke. 2015. Overview of current development in electrical energy storage technologies and the application potential in power system operation. Applied Energy 137 (Jan. 2015), 511-536. DOI : http://dx.doi.org/10.1016/j. apenergy.2014.09.081

[38] Adelina Madhja, Sotiris Nikoletseas, Dimitrios Tsolovos, and Alexandros A. Voudouris. 2018. Peer-to-Peer Energy-Aware Tree Network Formation. In Proceedings of the 16th ACM International Symposium on Mobility Management and Wireless Access (MobiWac'18). ACM, New York, NY, USA, 1-8. DOI : http://dx.doi.org/10.1145/3265863.3265875 event-place: Montreal, QC, Canada.

[39] Lone Malmborg, Ann Light, Geraldine Fitzpatrick, Victoria Bellotti, and Margot Brereton. 2015. Designing for Sharing in Local Communities. In Proceedings of the 33rd Annual ACM Conference Extended Abstracts on Human Factors in Computing Systems (CHI EA '15). ACM, New York, NY, USA, 2357-2360. DOI : http://dx.doi.org/10.1145/2702613.2702645 event-place: Seoul, Republic of Korea.

[40] Matthew Louis Mauriello, Brenna McNally, and Jon E. Froehlich. 2019a. Thermporal: An Easy-To-Deploy Temporal Thermographic Sensor System to Support Residential Energy Audits. In Proceedings of the 2019 CHI Conference on Human Factors in Computing Systems (CHI '19). ACM, New York, NY, USA, 113:1-113:14. DOI :

http://dx.doi.org/10.1145/3290605.3300343 event-place: Glasgow, Scotland Uk.

[41] Matthew Louis Mauriello, Chad Zanocco, Gregory Stelmach, June Flora, Hilary Boudet, and Ram Rajagopal. 2019b. An Energy Lifestyles Program for Tweens: A Pilot Study. In Extended Abstracts of the 2019 CHI Conference on Human Factors in Computing Systems (CHI EA '19). ACM, New York, NY, USA, LBW1118:1-LBW1118:6. DOI : http://dx.doi.org/10.1145/3290607.3312760 event-place: Glasgow, Scotland Uk.

[42] Arne Meeuw, Sandro Schopfer, Benjamin Ryder, and Felix Wortmann. 2018. LokalPower: Enabling Local Energy Markets with User-Driven Engagement. In Extended Abstracts of the 2018 CHI Conference on Human Factors in Computing Systems (CHI EA '18).
ACM, New York, NY, USA, LBW613:1-LBW613:6. DOI : http://dx.doi.org/10.1145/3170427.3188610 event-place: Montreal QC, Canada.

[43] Esther Mengelkamp, Johannes GÃd'rttner, Kerstin Rock, Scott Kessler, Lawrence Orsini, and Christof Weinhardt. 2018. Designing microgrid energy markets: A case study: The Brooklyn Microgrid. Applied Energy 210 (Jan. 2018), 870-880. DOI :

http://dx.doi.org/10.1016/j.apenergy .2017.06.054

[44] Thomas Morstyn, Niall Farrell, Sarah J. Darby, and Malcolm D. McCulloch. 2018. Using peer-to-peer energy-trading platforms to incentivize prosumers to form federated power plants. Nature Energy 3, 2 (Feb. 2018), 94-101. DOI:

http://dx.doi .org/10.1038/s41560-017-0075-y

[45] James Pierce and Eric Paulos. 2012. Beyond Energy Monitors: Interaction, Energy, and Emerging Energy Systems. In Proceedings of the SIGCHI Conference on Human Factors in Computing Systems (CHI'12). ACM, New York, NY, USA, 665-674. DOI :

http://dx.doi.org/10.1145/2207676.2207771 event-place: Austin, Texas, USA.

[46] Gareth Powells and Michael J. Fell. 2019. Flexibility capital and flexibility justice in smart energy systems. Energy Research \& Social Science 54 (Aug. 2019), 56-59. DOI :

http://dx.doi.org/10.1016/j.erss.2019.03.015

[47] Larissa Pschetz, Kruakae Pothong, and Chris Speed. 2019. Autonomous Distributed Energy Systems: Problematising the Invisible Through Design, Drama and Deliberation. In Proceedings of the 2019 CHI Conference on Human Factors in Computing Systems (CHI '19). ACM, New York, NY, USA, 387:1-387:14. DOI : http://dx.doi.org/10.1145/3290605.3300617 event-place: Glasgow, Scotland Uk.

[48] Giovanni Quattrone, Davide Proserpio, Daniele Quercia, Licia Capra, and Mirco Musolesi. 2016. Who Benefits from the "Sharing" Economy of Airbnb?. In Proceedings of the 25th International Conference on World Wide Web (WWW'16). International World Wide Web Conferences Steering Committee, Republic and Canton of Geneva, Switzerland, 1385-1394. DOI : http://dx.doi.org/10.1145/2872427.2874815 event-place: Montreal, Quebec, Canada.

[49] Sophia Ruester, Sebastian Schwenen, Carlos Batlle, and Ignacio Perez-Arriaga. 2014. From distribution networks to smart distribution systems: Rethinking the regulation of European electricity DSOs. Utilities Policy 31 (Dec. 2014), 229-237. DOI :

http://dx.doi.org/10.1016/j.jup.2014.03.007

[50] M. Sabounchi and J. Wei. 2017. Towards resilient networked microgrids: Blockchain-enabled peer-to-peer electricity trading mechanism. In 2017 IEEE Conference on Energy Internet and Energy System Integration (EI2). 1-5. DOI : http://dx.doi .org/10.1109/EI2 .2017.8245449 
[51] Nancy E. Schoenberg and Hege Ravdal. 2000. Using vignettes in awareness and attitudinal research. International Journal of Social Research Methodology 3, 1 (Jan. 2000), 63-74. DOI :

http://dx.doi.org/10.1080/136455700294932

[52] Will Simm, Maria Angela Ferrario, Adrian Friday, Peter Newman, Stephen Forshaw, Mike Hazas, and Alan Dix. 2015. Tiree Energy Pulse: Exploring Renewable Energy Forecasts on the Edge of the Grid. In Proceedings of the 33rd Annual ACM Conference on Human Factors in Computing Systems (CHI '15). ACM, New York, NY, USA, 1965-1974. DOI :

http://dx.doi.org/10.1145/2702123.2702285

event-place: Seoul, Republic of Korea.

[53] Brian J. Taylor. 2006. Factorial Surveys: Using Vignettes to Study Professional Judgement. The British Journal of Social Work 36, 7 (Oct. 2006), 1187-1207. DOI : http://dx.doi.org/10.1093/bjsw/bch345
[54] D. Vangulick, B. CornÃ l'lusse, and D. Ernst. 2018. Blockchain for Peer-to-Peer Energy Exchanges: Design and Recommendations. In 2018 Power Systems Computation Conference (PSCC). 1-7. DOI: http://dx.doi.org/10.23919/PSCC. 2018.8443042

[55] Jianing Zhai, Sid Chi-Kin Chau, and Minghua Chen. 2019. Stay or Switch: Competitive Online Algorithms for Energy Plan Selection in Energy Markets with Retail Choice. In Proceedings of the Tenth ACM International Conference on Future Energy Systems (e-Energy '19).

ACM, New York, NY, USA, 100-110. DOI : http://dx.doi.org/10.1145/3307772.3328287 event-place: Phoenix, AZ, USA.

[56] Chenghua Zhang, Jianzhong Wu, Chao Long, and Meng Cheng. 2017. Review of Existing Peer-to-Peer Energy Trading Projects. Energy Procedia 105 (May 2017), 2563-2568. DOI :

http://dx.doi.org/10.1016/j.egypro.2017.03.737 